\title{
Epistatic interactions between at least three loci determine the "rat-tail" phenotype in cattle
}

\author{
Jacqueline Knaust ${ }^{1}$, Frieder Hadlich ${ }^{1}$, Rosemarie Weikard ${ }^{1}$ and Christa Kuehn ${ }^{1,2^{*}}$
}

\begin{abstract}
Background: The "rat-tail" syndrome (RTS) is an inherited hypotrichosis in cattle, which is exclusively expressed in diluted coloured hair. The affected animals also suffer from disturbed thermoregulation, which impairs their health and growth performance. Phenotypic features that are similar to RTS are observed in dogs with black hair follicle dysplasia.
\end{abstract}

Results: We used a resource cross population between German Holstein and Charolais cattle breeds to prove that epistatic interactions between at least three independent genetic loci are required for the expression of the RTS phenotype. In this population, the RTS is exclusively expressed in animals with a eumelanic background that is due to the dominant $E^{D}$ allele at the melanocortin 1 receptor gene located on Bos taurus autosome (BTA) 18. In addition, only the individuals that are heterozygous at the dilution locus on BTA5 that corresponds to the premelanosome protein or silver gene variant c.64G>A were classified as displaying a RTS phenotype. Linkage and whole-genome association analyses using different models and different pedigrees allowed us to map a third locus (hereafter referred to as the RTS locus) that is essential for the expression of the RTS phenotype to the chromosomal region between 14 and 22 Mb on BTA5. Our findings clearly demonstrate that the RTS and dilution loci are distinct loci on BTA5.

Conclusions: Our study provides evidence that the RTS locus has effects on hair conformation and coat colour dilution and that the effect on coat colour dilution is clearly independent from that of the dilution locus. Finally, our results excluded several other loci that were previously reported to be associated with or to underlie hair conformation or pigmentation traits as the causal mutations of RTS and also several major functional candidate genes that are associated with hypotrichosis in humans. Our finding on the identification of a three-locus interaction that underlies RTS provides a prime example of epistatic interaction between several independent loci that is required for the expression of a distinct phenotype.

\section{Background}

Hypotrichosis is an inherited defect in mammals that is characterized by various degrees of sparse and curled malformed hair. In humans, a large number of causal mutations for hypotrichosis have been described [1]. The "rat-tail" syndrome (RTS) is a bovine congenital, inherited hypotrichosis that is characterized by misshaped, curly and sparse hair and by missing hairs at the tail

\footnotetext{
*Correspondence: kuehn@fbn-dummerstorf.de

${ }^{1}$ Institute for Genome Biology, Leibniz Institute for Farm Animal Biology,

18196 Dummerstorf, Mecklenburg-Vorpommern, Germany

Full list of author information is available at the end of the article
}

switch, which gave this defect its descriptive name [2] (Fig. 1). The defect in hair conformation is restricted to the pigmented areas of hair coat, and the affected animals suffer from disturbed thermoregulation, which impairs their health and growth performance [3]. RTS occurs in crosses between black cattle breeds (e.g., Angus and Holstein) and some European breeds that are characterized by colour dilution of the coat (e.g., Simmental, Charolais and Hereford). Few reports found in the literature describe single cases of congenital hypotrichosis that is restricted to the pigmented skin areas in Black and White Holstein cows $[4,5]$. The causal mechanism of this congenital malformation is unknown. 


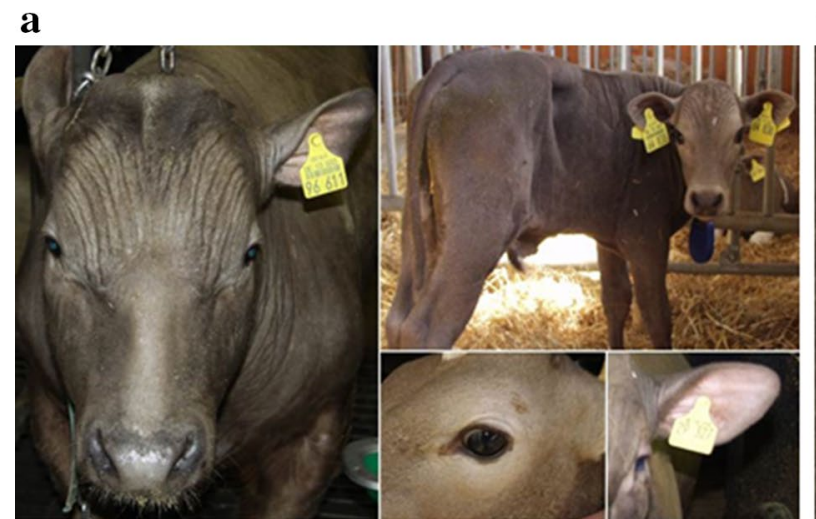

b

Fig. 1 Phenotype of animals with the "rat-tail" syndrome (RTS) within the SEGFAM population. a Extreme cases of hypotrichosis, scarce hair, skin folds are visible; eye lashes and hair in the ears are absent; affected animals often lack normal tail switch development. b RTS individuals show variation in hair structure and length between pigmented and non-pigmented areas

Schalles and Cundiff [3] postulated that RTS is caused by the epistatic interaction between two independent genetic loci. To date, affected cattle are assumed to carry an allele for black coat colour at one locus and to be heterozygous at a second locus. In Holstein and Angus cattle, the black coat colour is caused by the black $E^{\mathrm{D}}$ allele at the extension locus that corresponds to the melanocortin 1 receptor $(M C 1 R)$ gene on Bos taurus autosome (BTA) 18 ; allele $E^{\mathrm{D}}$ is dominant over the $E+$ wild-type allele and the recessive red $e$ allele $[6,7]$. There are several reports in the literature that indicate that mutations in the premelanosome protein (PMEL) gene [also known as silver gene (SILV) on BTA5] are responsible for the dilution locus and that this gene corresponds to the second locus involved in RTS $[8,9]$. However, in previous studies, we showed that neither mutations in the coding and regulatory regions of the $P M E L$ gene, nor splicing variants of this gene are associated with RTS $[10,11]$. Moreover, there are several reports on mutations that affect hair length and/or structure in Belted Galloway, Hereford and Fleckvieh cattle. Marron and Beever [12] and Markey [13] postulated that mutations in the hephaestin-like 1 (HEPHL1) gene on BTA29 and the keratin 71 (KRT71) gene on BTA5 are causal for variations in hair structure (HS) that are similar to RTS in Belted Galloway and Hereford, respectively. In addition, a recent study on the Fleckvieh breed reported a causal mutation in the keratin 27 (KRT27) gene on BTA19 for curly hair [14], which is a phenotype that has also been associated with RTS. However, it should be noted that these mutations affect both pigmented and non-pigmented hair, have no effect on hair length, and/or are present on a pheomelanic phenotypic background. These characteristics are clearly distinct from those of RTS. Thus, because of these partly controversial results, the complete genetic mechanism that underlies RTS is still under debate and our aim was to monitor the genetic architecture of RTS and to map one (or several) locus(i) that are involved in epistatic interaction in a German Holstein $\times$ Charolais $F_{2}$ cross population (i.e. SEGFAM population [15]), segregating for RTS.

\section{Methods}

Animals, traits and sampling

All experimental procedures were carried out according to the German animal care guidelines and were approved and supervised by the relevant authorities (Landesamt für Landwirtschaft, Lebensmittelsicherheit und Fischerei Mecklenburg-Vorpommern, Rostock; Landkreis Bad Doberan, Bad Doberan, Germany) of the State Mecklenburg-Vorpommern, Germany.

This study included individuals from the experimental resource cross population (SEGFAM) that was started in the $\mathrm{P}_{0}$ generation by crossing purebred German Holstein cows with purebred Charolais sires at the Leibniz Institute for Farm Animal Biology (FBN) [15]. This population was established by multiple ovulation and embryo transfer, which resulted in large half-sib and full-sib families. By crossing the dams from generation $F_{2}$ with German Holstein bulls, two large paternal half-sib backcross (BC) families were generated. For our analyses, we used 740 individuals from the $\mathrm{P}_{0}, \mathrm{~F}_{1}, \mathrm{~F}_{2}$ and $\mathrm{BC}$ generations. From the $\mathrm{BC}$ generation, only the calves with grey hair (genotype combination $E^{\mathrm{D}} / *, D \mathrm{c} / d \mathrm{c}+$ at the extension $(M C 1 R)$ [6] and dilution (PMELc.64G>A) loci, respectively [10]). For the different mapping designs, we analysed subsets of the full pedigree.

Since expression of the RTS phenotype varies among animals, for a precise dissection of the phenotype, all the animals were scored for hair conformation and 
pigmentation traits that are associated with RTS by double visual examination at 1 to 2 months of age (for the $F_{1}$, $\mathrm{F}_{2}$, and $\mathrm{BC}$ generations) and after puberty (at more than 15 months of age for the $F_{1}$ and $F_{2}$ generations) by two independent persons. The recorded traits were: degree of colour dilution of the coat in pigmented areas (hereafter termed Dilu for dilution), difference in hair length between pigmented and non-pigmented body areas (HLV for hair length variation), HS and hair density (HD) as well as an overall classification score (RT) taking into account all hair conformation traits associated with RTS. Differential scoring of RTS-associated traits was performed as described in Additional file 1: Figure S1.

For DNA genotyping, leukocytes were purified from blood samples and stored at $-20{ }^{\circ} \mathrm{C}$ until isolation of genomic DNA by phenol-chloroform standard methods. For the $\mathrm{P}_{0}$ and $\mathrm{BC}$ German Holstein sires, genomic DNA was extracted from sperm cells.

\section{Genotyping coat colour loci}

Genotypes at the extension locus were determined by sequencing a region of the corresponding $M C 1 R$ gene. First, a bovine-specific 444-bp MC1R sequence was amplified from genomic DNA by PCR using the following MC1R primers: MC1R F2: 5'-CCAGCCACCCTCCCCTTCACC-3' and MC1R R2: 5'-CGCAATGATCCTCCACGCTCG-3' that flank the causal mutations of alleles $E^{\mathrm{D}}$ (dominant black, eumelanin), $E+$ (wild type) and $e$ (recessive red, pheomelanin). PCR was performed as follows: an initial denaturation step at $95{ }^{\circ} \mathrm{C}$ for $2 \mathrm{~min}$, 12 cycles in a $2{ }^{\circ} \mathrm{C}$-touchdown protocol starting at an annealing temperature of $68^{\circ} \mathrm{C}\left(30 \mathrm{~s} 95{ }^{\circ} \mathrm{C}, 1 \mathrm{~min} 68^{\circ} \mathrm{C}\right.$, $\left.30 \mathrm{~s} 72{ }^{\circ} \mathrm{C}\right), 29$ cycles of amplification at $55^{\circ} \mathrm{C}\left(30 \mathrm{~s} 95^{\circ} \mathrm{C}\right.$, $1 \mathrm{~min} 55^{\circ} \mathrm{C}, 30 \mathrm{~s} 72{ }^{\circ} \mathrm{C}$ ) and finally an elongation step at $72{ }^{\circ} \mathrm{C}$ for 7 min using the GoTaq ${ }^{\circledR} \mathrm{G} 2$ Hot Start polymerase (Promega). Then, the PCR products were sequenced using the primer MC1R F1: 5'-TACTACTTTATCTGCTGCCTG-3' on a capillary sequencer (ABI 310, Applied Biosystems; MEGABACE, GE Healthcare) with BigDye ${ }^{\circledR}$ (Applied Biosystems) chemistry. Alleles were identified as described by Klungland et al. [6].

To identify the genotypes at the dilution locus [10], a region of the bovine $P M E L$ gene was amplified from genomic DNA, then digested with the restriction enzyme $B f m I$ (Fermentas), and alleles Dc (dominant diluted) and $d c+$ (recessive undiluted, wild type), which correspond to the PMEL c.64A and c.64G alleles were analysed as described in [10].

\section{Segregation analysis}

Segregation analysis of the RTS-associated traits was performed for 598 SEGFAM individuals from the $F_{2}$ and $\mathrm{BC}$ generations and for which scores for the five analyzed traits i.e. HS, HD, HLV, Dilu and RT, and genotypes at the extension and dilution loci were available. Four individuals were excluded from the final analysis because they carried an $E+$ allele at the extension locus, which is present at a low incidence within the population used.

\section{Whole-genome SNP genotyping}

Among the 740 SEGFAM animals, we genotyped all $\mathrm{P}_{0}$ sires, $\mathrm{F}_{1}$ and $\mathrm{F}_{2}$ individuals, and all grey $\mathrm{BC}$ calves $\left(E^{\mathrm{D}} / *, D \mathrm{c} / d \mathrm{c}+\right)$ and their German Holstein sires with the BovSNP50 v1/v2 Bead Chip (50k) or the BovLDv1 Bead Chip (6k) according to the manufacturer's instructions (Illumina Inc.).). After quality control using the Illumina Genome Studio v2011 (Illumina Inc.,) software and setting the following criteria: a call rate of at least $90 \%$ for the $50 \mathrm{k}$ and $95 \%$ for the $6 \mathrm{k}$ single nucleotide polymorphism (SNP) panels, a call frequency of at least $97 \%$, a GenTrain score of 0.60 and a minor allele frequency higher than 0.01, we retained 6802 (6k) and 37,218 (50k) SNPs for the linkage and association analyses, respectively. All genotypes were checked for Mendelian inconsistencies by using the PedCheck v1.1 program with levels 1 and 2 [16] and the PLINK v1.07 for IBD estimation [17].

\section{Linkage and association analysis}

First, we obtained a preliminary genomic localization of the RTS locus by genome-wide quantitative trait loci (QTL) analyses as described by Hanna et al. [18]. We fitted additive and additive-dominant linear regression models within the GRIDQTL software package [19] to identify regions that affect hair malformation i.e. for the scores for HS, HD, HLV, and RT. First, only sex was included as fixed effect to account for known differences in hair pigmentation between males and females. Subsequently, we also included the genotypes at the extension and dilution loci as fixed effects to account for potential interactions between the RTS locus and these two loci. Linkage analyses were conducted on the $6 \mathrm{k}$ genotyping data in $1 \mathrm{cM}$ steps based on the physical positions of SNPs according to the UMD3.1 assembly of the $B$. taurus genome [20] and the simplistic assumption that $1 \mathrm{cM} \sim 1 \mathrm{Mb}$ as in [18]. Chromosome- and genome-wide significance thresholds were determined by data permutations with 10,000 replicates and $95 \%$ confidence intervals were obtained based on 10,000 bootstrap samples. For the analyses using the $F_{2}$ full-sib families, we included the genotypes for generations $\mathrm{P}_{0}, \mathrm{~F}_{1}$ and $\mathrm{F}_{2}$ and the phenotypes for generation $\mathrm{F}_{2}$. For the linkage analyses using the $\mathrm{BC}$ half-sib families, we included the genotypes for generations $\mathrm{F}_{2}$ and $\mathrm{BC}$ and for the German Holstein sires that were used for backcrossing and the phenotypes for the $\mathrm{BC}$ generation. 
To verify our preliminary results, we conducted additional non-parametric linkage analyses using the MERLIN v1.1.2 software package (multipoint engine for rapid likelihood inference, [21]), based on the multipoint algorithm according to [22]. To this end, we selected the individuals with a eumelaneic dilute-coloured coat $\left(E^{\mathrm{D} / *}\right.$, $D c / d c+$ ) and rescored the animal phenotypes as "unaffected" (score 1), "affected" (scores 3 and 4) and "missing" (score 2, when the status was ambiguous) for the most discriminating RTS-associated trait, HLV. In our analyses, we used the exponential model of MERLIN [23] to test for linkage.

Finally, we performed a genome-wide association study (GWAS) using the SNP and Variation Suite v8 (Golden Helix, Inc., www.goldenhelix.com) to refine the localization of the RTS locus. For the GWAS, we selected the individuals from generations $\mathrm{F}_{1}, \mathrm{~F}_{2}$ and $\mathrm{BC}$ that fulfilled the precondition for RTS as determined by the segregation analysis, i.e. animals with at least one $E^{\mathrm{D}}$ allele at the extension locus and that were heterozygous for the $P M E L$ c.64G $>$ A locus. We calculated a genomic relationship matrix, which was included in a single locus mixed linear model (EMMAX, [24]) to account for relatedness and stratification effects within the dataset. Input variables were the scores for RT, HLV, HS, HD and Dilu. In addition to the linear mixed model, we also performed a case-control study, which required recoding of scores for RT, HLV and Dilu. For RT and HLV, all individuals were recoded essentially as described for the non-parametric linkage analyses. For the Dilu trait, since we had already excluded all individuals with a phenotype scored as 1 , we coded all animals with a score 2 as "affected", all animals with a score 4 as "unaffected" and all animals with a score 3 as "missing" for the case-control design. These recoded phenotypes were used for a case-control association study assuming an additive mode of inheritance and including 10 principal components to account for stratification effects. To account for multiple-testing in the linear mixed model as in the case-control study, we applied a Bonferroni correction of nominal $p$ values.

\section{Transcriptome analysis}

Skin samples were collected after slaughter from two piebald $\mathrm{F}_{2}$ bulls (one with RTS phenotype and one individual with wild type phenotype) from adjacent pigmented and non-pigmented areas of the neck essentially as described previously in [25]. In addition, we included pigmented skin samples from two other $F_{2}$ bulls with RTS phenotype to confirm the initial results. For each sample, the skin was trimmed from fat tissue and snap-frozen at $-80{ }^{\circ} \mathrm{C}$. RNA and library preparation for RNAseq using the Illumina Truseq RNA sample prep kit (Illumina Inc.,) was carried out as described in [25]. After quality control, the respective libraries were sequenced with a $2 \times 61$ bp paired-end protocol on an Illumina GAIIx sequencer. Quality control and alignment of reads including subsequent bioinformatic analyses were conducted as described in [25]. The resulting raw BAM files from the initial alignment of reads were visually screened for differences in the exon-intron structure of the genes that are located in the region between 10 and $25 \mathrm{Mb}$ on BTA5 and are known to be transcribed in the RTS and/or control animals. The gff3 file for the $B$. taurus annotation Release 104 of the bovine genome [26] and.gtf file [25] for project-specific gene annotation served as references for the genes that were located within the target region.

\section{Results}

\section{Segregation analysis and hypothesis}

The dataset used in this study included $50 \mathrm{P}_{0}, 69 \mathrm{~F}_{1}, 409 \mathrm{~F}_{2}$ and $212 \mathrm{BC}$ individuals from the SEGFAM cross population. A total of $598\left(393 \mathrm{~F}_{2}\right.$ and $205 \mathrm{BC}$ ) individuals with scores for each of the hair pigmentation and conformation traits were selected and used for the segregation analysis, which showed that a distinct RTS phenotype (RT scores of 3 and 4, Table 1) was restricted to individuals with the genotype combination $\left(E^{\mathrm{D} / *}, D \mathrm{c} / d \mathrm{c}+\right)$. However, among all $176 \mathrm{~F}_{2}$ and $205 \mathrm{BC}$ animals with this genotype combination, about $50 \%$ displayed a RTS phenotype ((94 and 124 with an RT score of 2 to 4 for the $\mathrm{F}_{2}$ and $\mathrm{BC}$ animals, respectively) and the remaining individuals displayed no indication on RTS phenotype. Two $F_{2}$ individuals had an RT score of 2 but were genotyped $E^{\mathrm{D}^{*}}, D \mathrm{c} / D \mathrm{c}$. No individual with a pheomelanic coat colour background (e/e at the extension locus) or with a "crème" or undiluted coat colour (homozygous for the mutant or wild type allele at the PMEL c.64G $>A$ locus) showed an RTS-associated hair conformation.

\section{Mapping the RTS locus by linkage analysis}

Initially, we obtained genome-wide significant signals on BTA5 for all hair conformation and pigmentation traits by using an additive-dominant model across all autosomes and the pseudoautosomal region of the sex chromosomes and by fitting sex as fixed effect within an $\mathrm{F}_{2}$ design that comprised $388 \mathrm{~F}_{2}$ individuals. Including genotypes from the $6 \mathrm{k}$ SNP panel consistently identified SNPs with high information content $(>0.95)$ across the whole genome (data not shown). The highest peaks of the test statistic were coincident with the position of the PMEL gene between 55 and $57 \mathrm{Mb}$ on BTA5 (see Additional file 2: Figure S2). However, the test statistic also showed a second peak exceeding the genome-wide significance threshold at $18 \mathrm{Mb}$. Based on the results from the segregation analysis, which had indicated that, in addition to the extension and dilution loci, a third locus 
Table 1 Segregation analysis of $598 \mathrm{~F}_{\mathbf{2}}$ and backcross (BC) SEGFAM calves based on the RTS classification score (RT)

\begin{tabular}{|c|c|c|c|c|c|c|c|c|}
\hline \multirow[t]{2}{*}{ Coat colour } & \multirow[t]{2}{*}{ MC1R } & \multirow[t]{2}{*}{ PMEL } & \multicolumn{3}{|l|}{$F_{2}$} & \multicolumn{3}{|l|}{$B C$} \\
\hline & & & Non-affected RT 1 & RT 3-4 & RT 2 & Non-affected RT 1 & RT 3-4 & RT 2 \\
\hline Black, no dilution & $E^{\mathrm{D}} / *$ & $d c+/ d c+$ & 40 & 0 & 0 & 0 & 0 & 0 \\
\hline Black, moderate dilution & $E^{\mathrm{D}} / *$ & $D c / d c+$ & 85 & 36 & 58 & 81 & 48 & 76 \\
\hline Black, strong dilution & $E^{\mathrm{D}} /^{*}$ & $D c / D C$ & 75 & 0 & 2 & 0 & 0 & 0 \\
\hline Red, no dilution & ele & $d c+/ d c+$ & 21 & 0 & 0 & 0 & 0 & 0 \\
\hline Red, moderate dilution & ele & $D c / d c+$ & 57 & 0 & 0 & 0 & 0 & 0 \\
\hline Red, strong dilution & e/e & $D c / D c$ & 22 & 0 & 0 & 0 & 0 & 0 \\
\hline
\end{tabular}

* Either allele $E^{\mathrm{D}}$ (dominant allele for black basic coat colour) or $e$ (recessive, red allele) at the extension (MC1R) locus; $d c+$ recessive wild type allele (no coat colour dilution) and Dc dominant allele (coat colour dilution) at the dilution (PMEL c.64G>A) locus

was involved in the mechanism underlying RTS, we subsequently performed a genome-wide linkage analysis by fitting an additive model with sex and the genotypes for $M C 1 R$ and PMEL c.64G>A as fixed effects. This analysis revealed a single locus with genome-wide $(\mathrm{q}<0.01)$ significant signals on BTA5, which affected all hair conformation traits (HS, HD, HLV, RT) (Fig. 2; Table 2). For all hair conformation traits, a single peak between 18 and $20 \mathrm{Mb}$ on BTA5 was observed, whereas two distinct peaks exceeding the genome-wide significance threshold were detected for Dilu at 18 and $57 \mathrm{Mb}$, respectively. A 2-QTL model confirmed that in addition to the PMEL c. $64 G>A$ locus, there were two segregating loci that were located on BTA5 and affected hair pigmentation dilution (F value for the 2-QTL model versus 1-QTL model:
37.46 (see Additional file 3: Figure S3). In addition to the genome-wide significant signal on BTA5, we detected chromosome-wide $(\mathrm{p}<0.05)$ significant signals using the $\mathrm{F}_{2}$ design for HLV and HS on BTA6 and 23, respectively, and for HS on BTA26 (Table 2).

The availability of two large $\mathrm{BC}$ half-sib groups that were generated from two German Holstein sires (97 and 109 BC calves from sires 1 and 2, respectively) allowed us to perform independent half-sib linkage analyses that tested the German Holstein sires for segregation of loci that affect RTS-associated hair conformation and pigmentation traits. Table 3 shows that the two sires did not share a chromosomal region that segregated for RTSassociated traits. A chromosome-wide significant locus that affects all hair conformation traits was detected on

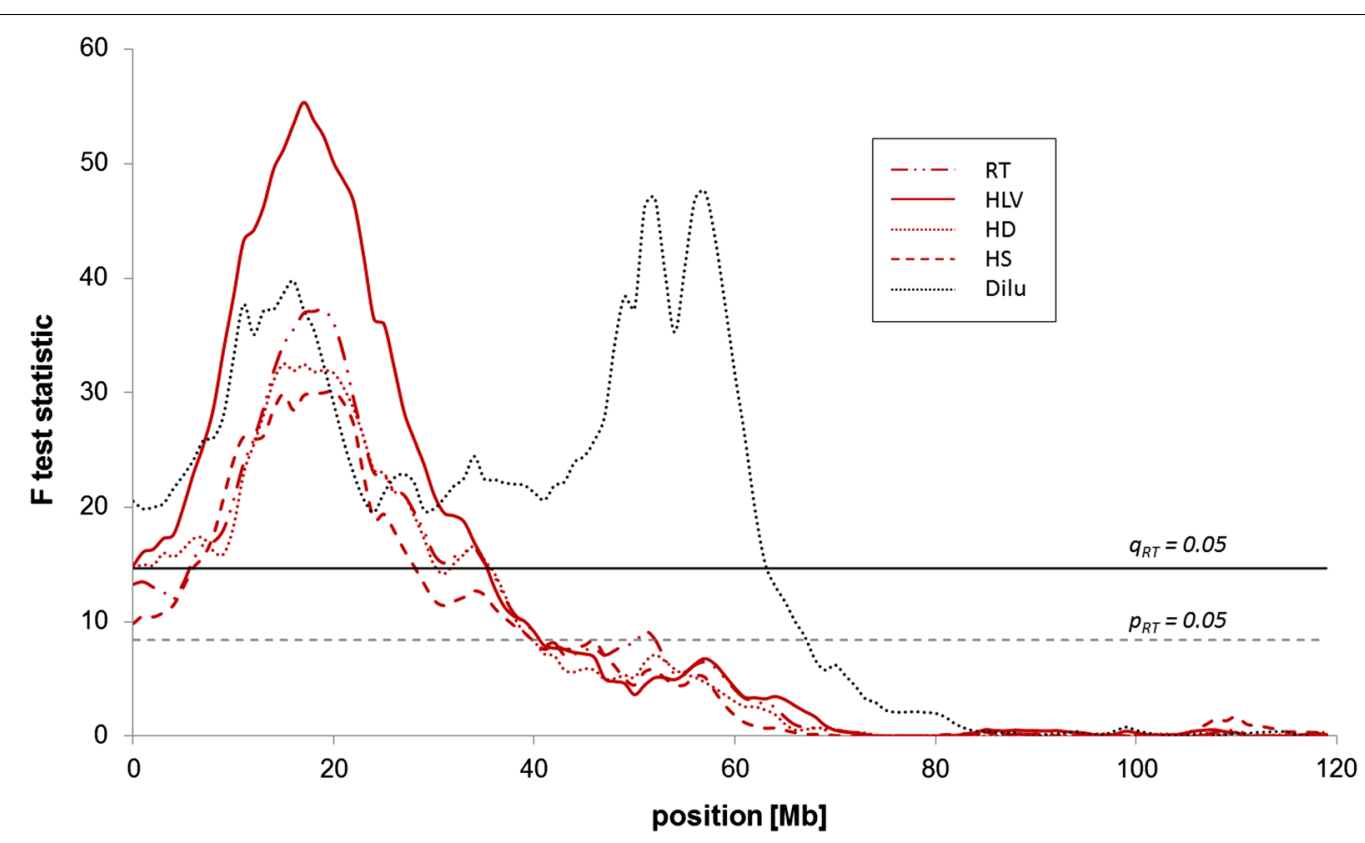

Fig. 2 F test statistic of the linkage analysis on BTA5 within the $F_{2}$ design. Dilu level of coat colour dilution in pigmented areas, HLV level of hair length variation between pigmented and non-pigmented coat, HS hair structure, HD hair density, RT RTS classification score phenotype 
Table 2 Results of the linkage analysis for RTS-associated hair pigmentation and pigmentation traits within the $F_{2}$ design

\begin{tabular}{|c|c|c|c|c|c|c|c|c|}
\hline \multirow[t]{2}{*}{ BTA } & \multirow[t]{2}{*}{ Trait } & \multirow[t]{2}{*}{ Position $^{a}$} & \multirow[t]{2}{*}{ F value } & \multirow[t]{2}{*}{$p$ value } & \multirow[t]{2}{*}{ q value } & \multirow[t]{2}{*}{$\mathrm{Cl}(\mathrm{Mb})$} & \multicolumn{2}{|l|}{ QTL effect } \\
\hline & & & & & & & Est. effect & SE \\
\hline 5 & Hair structure (HS) & 20 & 30.10 & $<0.0001$ & $<0.0001$ & $10.0-22.0$ & -0.48 & 0.09 \\
\hline 5 & Hair density (HD) & 15 & 32.51 & $<0.0001$ & $<0.0001$ & $7.0-27.0$ & -0.29 & 0.05 \\
\hline 5 & Hair lengths variation (HLV) & 17 & 55.37 & $<0.0001$ & $<0.0001$ & $11.0-22.0$ & -0.42 & 0.06 \\
\hline 5 & Degree of coat colour dilution (Dilu) & 57 & 47.60 & $<0.0001$ & $<0.0001$ & $11.0-57.0$ & 0.94 & 0.14 \\
\hline 5 & Degree of coat colour dilution (Dilu) & 16 & 39.75 & $<0.0001$ & $<0.0001$ & & & \\
\hline 5 & RTS classification phenotype (RT) & 19 & 37.21 & $<0.0001$ & $<0.0001$ & $14.0-22.0$ & -0.31 & 0.05 \\
\hline 6 & Hair lengths variation (HLV) & 71 & 10.32 & 0.0206 & ns & $21.0-112.0$ & $0-0.16$ & 0.05 \\
\hline 23 & Hair structure (HS) & 34 & 10.22 & 0.0123 & ns & $14.0-47.0$ & -0.24 & 0.07 \\
\hline 26 & Hair structure (HS) & 20 & 8.20 & 0.0307 & ns & $0.0-43.0$ & -0.22 & 0.08 \\
\hline
\end{tabular}

a Position in Mb; $\mathrm{p}$ value: chromosome-wide significance, q value: genome-wide significance, ns: not significant, $\mathrm{Cl}$ : $95 \%$ confidence interval in $\mathrm{Mb}$, Est. effect: estimated locus effects and the respective standard error (SE)

BTA 5 between 18 and $20 \mathrm{Mb}$ with the sire 1 family and confirmed the initial results obtained with the $\mathrm{F}_{2}$ design (Fig. 3). Interestingly, as with the $F_{2}$ design, two peaks were detected for the RT trait. It should be noted that sire 1 had an undiluted phenotype that is characteristic of Holstein black and white cattle. With the sire 1 family, a genome-wide significant locus for hair conformation traits (particularly HS) was detected on BTA10, and several chromosome-wide significant loci on BTA9, 19, 25, 26 and 29, in addition to the locus on BTA5 for RTSassociated traits. With the sire 2 family, a genome-wide significant locus for RT on BTA20 (see Additional file 4: Figure S4) and several chromosome-wide significant loci for one or more RTS-associated traits on BTA3, 9, 13, 14, 18 , and 23 were detected. On BTA18, the extension locus was more than $30 \mathrm{Mb}$ away from the region that segregated for Dilu and HLV and outside the confidence interval for Dilu. Concordant genomic mapping of several loci for different hair conformation traits at the same position suggested that some genes that impact variation in hair traits may have pleiotropic effects.

Additional non-parametric analyses using MERLIN were performed using $F_{1}$ and $F_{2}$ individuals and showed significant signals on BTA5 at $15 \mathrm{Mb}$ for the most discriminating hair conformation trait $\operatorname{HLV}(\mathrm{q}<0.01$, after accounting for testing 30 chromosomes) and confirmed the initial results from the linear regression QTL analysis.

\section{Genome-wide association study}

We performed a GWAS to further narrow down the localization of the locus/loci that underlie RTS. When only grey $\left(E^{\mathrm{D} / *}, D \mathrm{c} / d \mathrm{c}+\right) \mathrm{F}_{1}, \mathrm{~F}_{2}$ and $\mathrm{BC}$ individuals were included, genome-wide significant signals were detected on BTA5 with the linear mixed model as well as with the case-control design, which confirmed the results of the initial linkage analyses (Fig. 4; Additional file 5: Figure S5). With the mixed linear model and using the non-recoded phenotype scores, we obtained genome-wide significant associations for HLV and RT (Table 4). SNP BTA74302-no-rs on BTA5 (located at 18,047,140 bp) was the most significant SNP for both traits. In the case-control design, we obtained genome-wide significant results for HLV, RT and Dilu, and SNP BTA-74302-no-rs was again highly significant with the lowest q value of all SNPs for HLV and was among the three markers with the most significant associations for RT and Dilu in our GWAS. This SNP had contrasted allele frequencies in the Holstein and Charolais $\mathrm{P}_{0}$ founder animals i.e. frequency of allele $1_{\text {Charolais }}=0.90$ and frequency of allele $1_{\text {Holstein }}=0.32$ ). In spite of its significant association signals, this SNP was not fully associated with RTS (see Additional file 6: Table $\mathrm{S} 1$ ). The SNP that showed the most significant association with RT and Dilu was Hapmap44614-BTA-72802 at position $21,114,508$ bp on BTA5. For all trait-model combinations, except the mixed linear model for HLV, the three SNPs with the strongest genome-wide significance were located in the interval between 18.04 and $21.11 \mathrm{Mb}$. This position is within the confidence intervals for the $R T S$ locus that were determined by linkage analysis with the $\mathrm{F}_{2}$ and the $\mathrm{BC}$ subsets.

After the effects of the extension and dilution loci were fitted, including a dominant effect in the model did not increase the test statistic compared to that of an additive effect in the linkage or the association analyses for RT, HLV, HD or KRT. The only genome-wide significant association $(\mathrm{q}=0.005)$ that was detected in the dominant mixed model association analysis was between Dilu and SNP Hapmap27767-BTA-154179 located at $60,263,049$ bp on BTA5.

\section{Transcriptome analysis}

In the region between 10 and $25 \mathrm{Mb}$ on BTA5, 102 genes are annotated in Release 104 of the B. taurus genome assembly. For all the samples investigated by RNAseq, 
Table 3 Results of the linkage analysis for RTS-associated hair pigmentation and pigmentation traits within a half-sib backcross (BC) design for each of the two sire sib ships

\begin{tabular}{|c|c|c|c|c|c|c|c|c|}
\hline \multirow[t]{2}{*}{ BTA } & \multirow[t]{2}{*}{ Trait } & \multirow[t]{2}{*}{ Position $^{a}$} & \multirow[t]{2}{*}{ F value } & \multirow[t]{2}{*}{$\mathrm{p}$ value } & \multirow[t]{2}{*}{ q value } & \multirow[t]{2}{*}{$\mathrm{Cl}(\mathrm{Mb})$} & \multicolumn{2}{|c|}{ Locus effect } \\
\hline & & & & & & & Est. effect & SE \\
\hline 3 & Hair length variation (HLV) & 83 & 9.35 & 0.0303 & ns & $13.0-113.02$ & -0.63 & 0.20 \\
\hline 5 & Hair structure (HS) & 20 & 10.93 & 0.0130 & ns & $8.0-105.51$ & -1.01 & 0.31 \\
\hline 5 & Hair density (HD) & 18 & 14.80 & 0.0033 & ns & $5.0-63.01$ & -0.66 & 0.18 \\
\hline 5 & Hair length variation (HLV) & 18 & 11.42 & 0.0126 & ns & $4.0-80.01$ & -0.70 & 0.21 \\
\hline 5 & Level of coat colour dilution (Dilu) & 18 & 11.17 & 0.0116 & ns & $10.0-116.01$ & 0.38 & 0.12 \\
\hline 5 & RTS classification phenotype (RT) & 49 & 13.16 & 0.0072 & ns & $15.0-65.01$ & -0.67 & 0.18 \\
\hline 8 & Hair structure (HS) & 44 & 10.49 & 0.0210 & ns & $8.0-107.01$ & -0.99 & 0.30 \\
\hline 8 & Hair length variation (HLV) & 38 & 10.25 & 0.0201 & ns & $4.0-101.01$ & -0.69 & 0.21 \\
\hline 8 & RTS classification phenotype (RT) & 13 & 9.01 & 0.0366 & ns & $8.0-94.01$ & -0.56 & 0.19 \\
\hline 9 & Hair structure (HS) & 90 & 9.20 & 0.0205 & ns & 57.0-98.02 & 0.74 & 0.25 \\
\hline 9 & Hair density (HD) & 98 & 7.97 & 0.0371 & ns & $50.0-103.02$ & 0.56 & 0.20 \\
\hline 9 & Hair length variation (HLV) & 90 & 10.24 & 0.0147 & ns & $35.0-96.02$ & 0.64 & 0.20 \\
\hline 9 & RTS classification phenotype (RT) & 98 & 8.42 & 0.0326 & ns & $41.0-101.02$ & 0.50 & 0.17 \\
\hline 10 & Hair structure (HS) & 96 & 18.08 & 0.0011 & 0.0207 & 10.0-98.01 & 1.24 & 0.29 \\
\hline 10 & Hair density (HD) & 96 & 12.48 & 0.0076 & ns & $39.0-103.01$ & 0.60 & 0.17 \\
\hline 10 & RTS classification phenotype (RT) & 94 & 10.51 & 0.0160 & ns & $0.0-103.01$ & 0.61 & 0.19 \\
\hline 13 & Hair density (HD) & 58 & 14.53 & 0.0026 & ns & $23.0-62.02$ & 0.73 & 0.19 \\
\hline 13 & RTS classification phenotype (RT) & 42 & 12.47 & 0.0067 & ns & $23.0-70.02$ & 0.59 & 0.17 \\
\hline 14 & RTS classification phenotype (RT) & 36 & 9.80 & 0.0170 & ns & $13.0-75.02$ & -0.53 & 0.17 \\
\hline 18 & Hair structure (HS) & 46 & 10.72 & 0.0104 & ns & $0.0-51.02$ & 0.80 & 0.25 \\
\hline 18 & Hair length variation (HLV) & 43 & 14.55 & 0.0030 & ns & $12.0-48.02$ & 0.76 & 0.20 \\
\hline 18 & Level of coat colour dilution (Dilu) & 46 & 15.19 & 0.0020 & ns & $40.0-56.02$ & -0.39 & 0.10 \\
\hline 19 & Hair structure (HS) & 8 & 9.42 & 0.0250 & ns & $7.0-53.01$ & 0.95 & 0.31 \\
\hline 20 & Hair density (HD) & 70 & 14.05 & 0.0025 & ns & $44.0-70.02$ & -0.71 & 0.19 \\
\hline 20 & RTS classification phenotype (RT) & 57 & 19.33 & 0.0003 & 0.009 & $46.5-70.02$ & -0.71 & 0.16 \\
\hline 23 & RTS classification phenotype (RT) & 6 & 9.07 & 0.0192 & ns & $3.0-51.02$ & 0.51 & 0.17 \\
\hline 25 & Hair structure (HS) & 5 & 10.25 & 0.0127 & ns & $0.0-36.01$ & 0.98 & 0.31 \\
\hline 25 & Hair density (HD) & 5 & 8.29 & 0.0265 & ns & $2.0-39.01$ & 0.51 & 0.18 \\
\hline 25 & Hair length variation (HLV) & 5 & 10.19 & 0.0108 & ns & $1.0-36.01$ & 0.67 & 0.21 \\
\hline 25 & RTS classification phenotype (RT) & 4 & 9.00 & 0.0194 & ns & $0.0-40.01$ & 0.57 & 0.19 \\
\hline 26 & Hair density (HD) & 36 & 9.01 & 0.0220 & ns & $0.0-48.01$ & -0.53 & 0.18 \\
\hline 29 & Hair density (HD) & 47 & 8.71 & 0.0226 & ns & $0.0-49.01$ & -0.52 & 0.18 \\
\hline 29 & RTS classification phenotype (RT) & 47 & 10.51 & 0.0124 & ns & $3.0-49.01$ & -0.60 & 0.19 \\
\hline
\end{tabular}

a Position in Mb; $\mathrm{p}$ value: chromosome-wide significance, q value: genome-wide significance, ns: not significant, Sire: sire family significantly segregating for the respective locus, $\mathrm{Cl}$ : 95 \% confidence interval in $\mathrm{Mb}$, Est. effect: estimated locus effects and the respective standard error (SE)

more than 35 million uniquely mapped fragments were available for whole-transcriptome data analysis. Genes with at least ten aligned reads were assigned the status "expressed". From the 102 genes, 37 exceeded this threshold (see Additional file 7: Table S2), but none were expressed exclusively in the skin of animals with RTS or in wild type pigmented or unpigmented skin. In addition, although we observed alternative splicing for several genes, no RTS-associated alternative transcript isoforms were observed for any of the genes between RTS and wild type pigmented skin (see Additional file 7: Table S2). Transcripts for the POC1 centriolar protein B (POC1B) and FYVE, RhoGEF and PH domain containing 6 (FGD6) genes were detected in one pigmented RTS skin that differed from wild type skin, but this was not consistent across all three pigmented RTS samples.

\section{Discussion}

Segregation of RTS is present within the experimental cross SEGFAM [15] population between black German Holstein and Charolais breeds. At the extension locus, black German Holstein cattle carry at least one dominant black allele $\left(E^{\mathrm{D}}\right)$, whereas Charolais are homozygous $(e / e)$ for the recessive allele. However, due to homozygosity of 


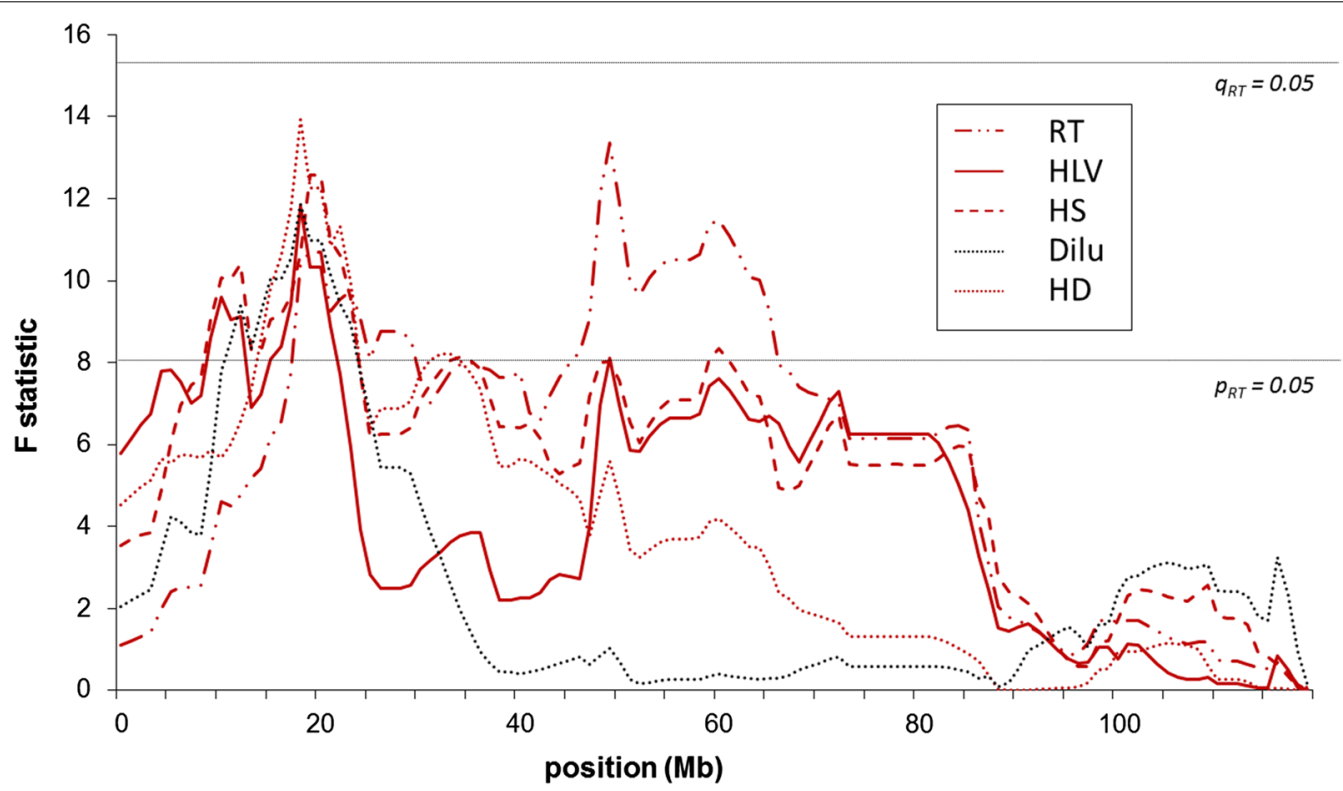

Fig. 3 Test statistic of the linkage analysis on BTA5 in the half-sib sire 1 family. Dilu level of coat colour dilution in pigmented areas, HLV level of hair length variation between pigmented and non-pigmented coat, HS hair structure, HD hair density, RT RTS classification score phenotype

the mutant $D c$ allele at the dilution locus, Charolais cattle express an extremely diluted coat colour ("crème") instead of the red undiluted pigmentation [10]. The homozygous recessive wild type genotype $(d c+/ d c+)$ that is present in German Holstein cattle causes an undiluted basic coat colour. By crossing these two breeds, offspring with the genotype combination $E^{\mathrm{D}} / *, D \mathrm{c} / d \mathrm{c}+$ are generated, which agrees with the initial hypothesis of a two-locus model i.e. the extension and dilution loci as the causal mechanism for RTS phenotypes described by Schalles and Cundiff [3]. However, our results from the segregation analysis prove that this two-locus model is not sufficient to fully explain the RTS phenotype. Although RTS obviously requires the expression of a diluted eumelanic pigmentation background (Table 1) that fits with the initial hypothesis of Schalles and Cundiff [3], our data provide evidence that epistatic interaction with at least a third independent locus is required for the expression of the RTS phenotype. This finding contrasts with reports about causal mutations for RTS within the PMEL locus [8,9]. Our data based on linkage and association analyses also support the conclusions from the segregation analysis. Both linkage analysis with the full-sib $\mathrm{F}_{2}$ and half-sib $\mathrm{BC}$ designs and association analysis with a population comprising $F_{1}, F_{2}$ and BC animals, mapped RTS-associated hair conformation traits between 15 and $20 \mathrm{Mb}$ on BTA5, which is far from the PMEL gene at $57.6 \mathrm{Mb}$. PMEL is outside the confidence interval for RTS-associated traits as determined by linkage analysis bootstrapping. Correspondingly, the genotype combination of $E^{\mathrm{D} / *}, D \mathrm{c} / d \mathrm{c}+$ is required, but not sufficient for the expression of the RTS phenotype. Thus, we postulate the existence of a third locus that plays a role in the RTS phenotype and is in epistatic interaction with the extension and dilution loci. Otherwise all or almost all grey individuals with the genotype combination $E^{\mathrm{D}} / *, D \mathrm{c} / d \mathrm{c}+$ at the extension and dilution loci would display the RTS phenotype. An autosomal additive model of inheritance for RTS is suggested, because after fitting the effects of the extension and dilution loci, including a dominant effect did not increase the test statistic in the linkage or association analyses for RTS-associated traits except for Dilu.

In dogs, black hair follicular dysplasia (BHFD) is an inherited recessive defect that also specifically affects pigmented hair in piebald individuals [27]. BHFD has a recessive mode of inheritance and is characterized by coat colour dilution, hypotrichosis and hair conformation defects. BHFD is assumed to be identical to the coat colour dilution (CDA) defect observed in some nonpiebald breeds with dilute coat colour (e.g., Pinschers). Interestingly, Welle et al. [28] also postulated that, in addition to a gene causing coat colour dilution, another factor was required for the expression of the CDS/BHFD phenotype. While for several dog breeds with BHFD/ CDA, melanophilin $(M L P H)$ was identified as the causative gene for coat colour dilution [29], in cattle, mutations in the $P M E L$ gene are causal for coat colour dilution and represent one epistatic factor for RTS.

By using several designs (full sib $\mathrm{F}_{2}$, half-sib $\mathrm{BC}$, joint $\mathrm{F}_{1}, \mathrm{~F}_{2}$, and $\mathrm{BC}$ populations) and statistical analyses 

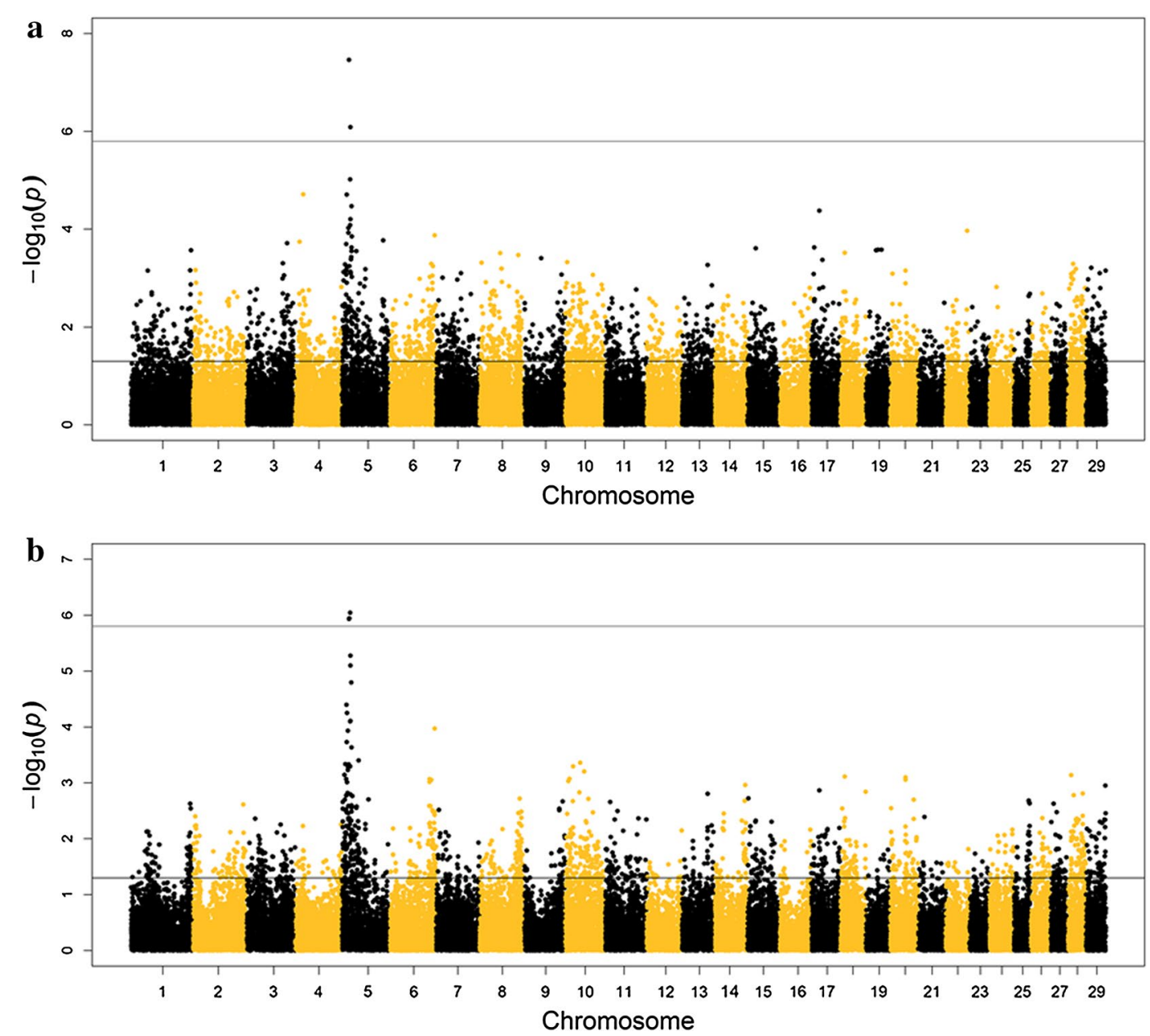

Fig. 4 Manhattan plot of the whole-genome association analysis for RTS classification score (RT) phenotype. a Fitting a single-locus mixed model (MM) including a genomic relationship matrix. b Case-control study (CC) for the trait RTS classification score (RT) phenotype. The upper horizontal line represents the genome-wide significance threshold $q=0.05$, the lower horizontal line represents the nominal $p$ value $=0.05$

(linkage and association mapping), we were able to show that the third epistatic locus involved in RTS (in addition to the extension and dilution loci) mapped to BTA5 between 14 and $22 \mathrm{Mb}$. This genuine RTS locus obviously affects HS as well as coat colour dilution (Table 2). The hypothesis of a third locus is supported by the results of the GWAS, which included only animals with a heterozygous genotype at the PMEL c.64G>A locus and showed a significant association between Dilu and SNP BTA74302-no-rs. This association signal was located exactly at the same position as the locus for RTS-associated hair conformation traits. Further evidence for more than one locus affecting coat colour dilution was provided, because fitting the PMEL c.64G>A genotype as a fixed effect into the linkage analysis did not fully eliminate a highly significant peak in the test statistic on BTA5 for coat colour dilution in the $\mathrm{F}_{2}$ design, which had been obtained in a model without this fixed effect. Instead, two individual peaks at 16 and $57 \mathrm{Mb}$ were observed in the extended model with the PMEL c.64G>A genotype as a fixed effect. This indicates that the RTS locus located between 14 and $22 \mathrm{Mb}$ also affects coat colour dilution and that the chromosomal region that includes the $P M E L$ gene contains information in addition to the PMEL c.64.G>A mutation that affects coat colour dilution.

When we first performed linkage analyses without fitting the genotypes at the PMEL c.64G>A and extension loci into the model, we obtained highly significant peaks for all monitored RTS-associated traits that were located on BTA5 between 55 and $57 \mathrm{Mb}$ (see Additional file 2: Figure S2), which corresponds exactly to the localization of the PMEL gene. Thus, our data show that missing an essential factor in an epistatic interaction network will yield false positive results. We hypothesize that previous 
Table 4 Summary of genome-wide significant associations from a GWAS for hair conformation and hair dilution traits associated with the RTS in $F_{1}, F_{2}$ and $B C$ individuals with at least one $E^{D}$ allele at the extension locus and heterozygous for the PMEL C.64G>A locus

\begin{tabular}{|c|c|c|c|c|c|c|}
\hline Trait & Model $^{\mathrm{a}}$ & Marker & BTA & Position (bp) & $p$ value & q value ${ }^{b}$ \\
\hline Level of coat colour dilution & Case-control & Hapmap44614-BTA-72802 & 5 & $21,114,508$ & 8.90E-07 & 0.033 \\
\hline Level of coat colour dilution & Case-control & Hapmap53597-rs29010787 & 5 & $19,015,523$ & $1.15 E-06$ & 0.043 \\
\hline Level of coat colour dilution & Case-control & BTA-74302-no-rs & 5 & $18,047,140$ & $1.22 \mathrm{E}-06$ & 0.046 \\
\hline Hair length variation & Case-control & BTA-74302-no-rs & 5 & $18,047,140$ & $1.74 \mathrm{E}-07$ & 0.007 \\
\hline Hair length variation & Case-control & Hapmap42402-BTA-103350 & 5 & $21,484,201$ & $2.69 E-07$ & 0.010 \\
\hline Hair length variation & Case-control & Hapmap44614-BTA-72802 & 5 & $21,114,508$ & $1.04 \mathrm{E}-06$ & 0.039 \\
\hline Hair length variation & Case-control & ARS-BFGL-NGS-26310 & 5 & $12,123,561$ & $1.17 \mathrm{E}-06$ & 0.044 \\
\hline RTS classification phenotype & Case-control & Hapmap44614-BTA-72802 & 5 & $21,114,508$ & $9.03 E-07$ & 0.033 \\
\hline RTS classification phenotype & Case-control & Hapmap53597-rs29010787 & 5 & $19,015,523$ & $1.14 \mathrm{E}-06$ & 0.043 \\
\hline RTS classification phenotype & Case-control & BTA-74302-no-rs & 5 & $18,047,140$ & $1.17 \mathrm{E}-06$ & 0.044 \\
\hline Hair length variation & Mixed & BTA-74302-no-rs & 5 & $18,047,140$ & 7.36E-09 & 0.0003 \\
\hline Hair length variation & Mixed & ARS-BFGL-NGS-26310 & 5 & $12,123,561$ & $1.68 \mathrm{E}-07$ & 0.006 \\
\hline Hair length variation & Mixed & BTA-103542-no-rs & 5 & $16,795,347$ & $1.84 \mathrm{E}-07$ & 0.007 \\
\hline RTS classification phenotype & Mixed & BTA-74302-no-rs & 5 & $18,047,140$ & $3.43 E-08$ & 0.001 \\
\hline RTS classification phenotype & Mixed & Hapmap42402-BTA-103350 & 5 & $21,484,201$ & $8.16 \mathrm{E}-07$ & 0.030 \\
\hline
\end{tabular}

a Case-control: genotype association study in a case-control design, Mixed: mixed linear models including phenotype scores

b Bonferroni corrected $\mathrm{p}$ values to account for multiple testing

reports on the role of $P M E L$ in the mechanism that underlies RTS $[8,9]$ may have suffered from this problem.

Our data from the linkage analyses on the $\mathrm{F}_{2}$ and $\mathrm{BC}$ populations also suggest that there are additional loci, which combined with the extension and dilution loci, cause hair conformation modifications. Genome-wide significant effects on HS and RTS classification score (RT) from loci on BTA10 and 20 that were detected by using single large half-sib families provide support for this hypothesis. To our knowledge, none of these loci have been detected in previous studies. However, we did not observe any significant linkage or association signal nearby the HEPHL1 gene on BTA29 or the KRT71 gene on BTA5, two genes which had been reported to harbour mutations responsible for variations in hair length or structure that share phenotypic similarities with RTS. These results suggest that there might be a substantial amount of genetic heterogeneity in phenotypes with "rat-tail"-like features. Furthermore, it demonstrates that a very detailed description of the hair phenotype is required to identify the respective causal mutations.

The keratin gene cluster is a very obvious functional candidate region located on BTA5. However, the position of this cluster is outside the confidence interval for RTS as determined by linkage analysis. In addition, no SNP located within the keratin cluster was associated with the overall "rat-tail" phenotype RT ( $p$ value $<0.05$ ). Thus, mutations within the keratin gene cluster are not likely causal mutations for RTS. Furthermore, all genes reviewed by Basit et al. [30] as causal for hypotrichosis in humans, were excluded as candidates for RTS based on positional data from our linkage and association studies: none of our models or designs indicated a significant locus in one of the respective orthologous bovine chromosomal regions.

Regarding potential candidate genes for RTS, the KITLG (ligand for the receptor-type protein-tyrosine kinase KIT) gene located at $18 \mathrm{Mb}$ on BTA5 is a very obvious candidate based on its known role in pigmentation [31, 32]. However, sequencing the coding region and the proximal promoter region of the KITLG gene did not yield any variant associated with RTS (data not shown). This does not preclude that a regulatory region of the KITLG gene may contain a causal mutation for RTS, as demonstrated by Guenther et al. [33]. These authors showed that, in humans, a SNP that is located $350 \mathrm{~kb}$ upstream of the KITLG transcription start site, significantly alters the activity of a hair follicle associated enhancer and causes blond hair in North Europeans. Results from RNAseq-based holistic transcriptome analysis did not reveal any changes in the exon-intron structure of the currently annotated genes, which are located in the interval between 10 and $25 \mathrm{Mb}$ on BTA5 and are expressed in the skin, between individuals with RTS and wild type cattle. However, we identified a number of exons in genes that have not yet been annotated but are expressed in pigmented and/or unpigmented skin. This demonstrates the need for further efforts to 
improve the functional genome annotation in cattle. Interestingly, Basit et al. [34] described a digenic mode of inheritance for hypotrichosis in two independent human families with one locus represented by mutations in the cadherin $3(\mathrm{CDH} 3)$ gene and a second locus located in a chromosomal region (HSA12q21.2-22) that is syntenic with a region on BTA5 around $18 \mathrm{Mb}$. As in our study, the authors did not identify any defect-associated mutation in the coding region or splice sites of six functional candidate genes including KITLG on HSA12q21.2-22.

\section{Conclusions}

Our study provides evidence that the RTS phenotype results from an epistatic interaction between three independent loci: dilution (that corresponds to the PMEL gene at $55 \mathrm{Mb}$ on BTA5), extension (that corresponds to the MC1R gene on BTA18) and the RTS locus that is located in the interval between 14 and $22 \mathrm{Mb}$ on BTA5. The prerequisites for expression of the RTS phenotype are a eumelanic background due to the presence of the dominant $E^{\mathrm{D}}$ allele at $M C 1 R$ (extension locus) and a heterozygous genotype at the $P M E L$ gene variant $c .64 G>A$ (dilution locus). The positions of the RTS and dilution loci on BTA5 are clearly distinct. Finally, our results excluded several potential loci that were reported to be associated with RTS or that underlie hair conformation or pigmentation traits as the causal mutations of RTS and also several major functional candidate genes that are associated with hypotrichosis in humans. Additional studies are required to investigate whether RTS is caused by a mutation in a yet unknown functional element located in the target chromosomal region on BTA5 and to analyze the substantial genetic heterogeneity of the RTS phenotype. However, our finding on the identification of a three-locus interaction that underlies RTS provides a prime example of epistatic interaction between several independent loci that is necessary for the expression of a distinct phenotype.

\section{Additional files}

Additional file 1: Figure S1. Phenotype scoring of RTS associated hair conformation and pigmentation traits. Phenotypes and corresponding scores for HS: HS 1: wild type, straight hair, HS 2: hair with fluffy, smooth structure, HS 3: curly hair with a mildly frizzy/wiry structure, HS 4: coarse, frizzy/wiry hair, HS 5: very coarse/wiry hair, HS 6: extremely coarse/wiry HS. Phenotypes and corresponding scores for hair density (HD): HD 1: wild type, full, thick, long hair covering the whole body as well as in the ears and around the eyes, HD 2: scarce hair at the trunk and/or few, short hair in the ears and around the eyes, HD 3: short and sparse hair in the ears and around the eyes as well as thin coat, HD 4: without eye lashes and/or hair in the ears and with very thin hair coat and/or bald areas. Phenotypes and corresponding scores for hair length variations between pigmented and unpigmented areas of spotted animals (HLV): HLV 1: wild type, equally long hair in pigmented and unpigmented sections. HLV 2: pigmented hair mildly shorter than unpigmented hair, HLV 3: pigmented hair moderately shorter than unpigmented hair, HLV 4: pigmented hair extremely shorter than unpigmented hair. Animals without white spotting marks were scored according to their phenotype for RT. Phenotypes and corresponding scores for RTS classification phenotype: RT 1: wild type without any RTS-associated hair conformation malformation, RT 2: mild HLV and/ or short hair in ears and short, thin eye lashes, reduced tail switch. RT 3: sparse, coarse hair, moderate HLV, few hairs in ears/around eyes and/or few hair in eye lashes, tail switch only rudimentary. RT 4: very sparse coat, extreme HLV, scarce/wiry hair in pigmented areas, no hair in ears, barely/ no eyelashes, extremely visible skin folds at face/throat, bald areas surrounding the eyes/in the ears, missing tail switch. Status of tail switch was only informative, if the end of the tail was pigmented. Phenotypes and corresponding scores for coat colour dilution (Dilu) in pigmented coat sections on a eumelanic (black, brown) and pheomelanic (red, yellow) colour background. Dilu 1: undiluted coat colour, black or red-brown; Dilu 2: mild colour dilution with dark grey colour for the black/brown background and orange/caramel for the red background, Dilu 3: mouse grey/ bright grey (black background) or yellow/beige (red background), Dilu 4: extremely diluted, with crème and nearly white pigmentation.

Additional file 2: Figure S2. Test statistic for hair conformation and pigmentation traits on BTA5 from the $F_{2}$ full sib design fitting sex as fixed effect in an additive-dominant model. HLV: level of hair length variation between pigmented and unpigmented body areas, HS: hair structure, HD: hair density, RT: RTS classification phenotype. The test statistic for Dilu is not shown due to excessively high F values $(>500)$, which exceed the scale of the $y$ axis.

Additional file 3: Figure S3. Contour plot showing the test statistic for coat colour dilution (Dilu) on BTA5 for a 2-QTL model in a $F_{2}$ full sib design. The $x$ and $y$ axes represent chromosomal positions for QTL1 and QTL2, respectively. The $z$ axis indicates the F value of the QTL test statistic for a 2-QTL model.

Additional file 4: Figure S4. Test statistic of the linkage analysis on BTA20 for the half-sib family sire 2. Dilu: level of coat colour dilution in pigmented areas, HLV: level of hair lengths variation between pigmented and unpigmented coat, HS: hair structure, HD: hair density, RT: RTS classification phenotype.

Additional file 5: Figure S5. Manhattan plots of a whole-genome association study for the traits: hair length variation (HLV), hair structure (HS), hair density (HD) and coat colour dilution (Dilu) in the linear mixed model and for coat colour dilution (Dilu) and hair length variation (HLV) in the case-control design. The upper horizontal line represents the genomewide significance threshold $q=0.05$, the lower horizontal line represents the nominal $p$ value $=0.05$. Only $F_{1}, F_{2}$ and $B C$ individuals with $a E^{D} /{ }^{*}$ and $D c / d c+$ genotype at the extension and the dilution locus were included.

Additional file 6: Table S1. Genotypes of wild type and RTS-affected $F_{2}$ individuals for SNP BTA-74302-no-rs. $F_{2}$ wt: $F_{2}$ individual with wild type phenotype and $F_{2}-R T: F_{2}$ individual with RTS phenotype.

Additional file 7: Table S2. Genes in the region between 10 and $25 \mathrm{Mb}$ on BTA5 that were expressed in the pigmented skin transcriptome of RTSaffected and wild type animals.

\section{Authors' contributions}

JK carried out phenotyping, molecular genetic analyses and linkage analyses, participated in bioinformatics analyses, association studies and drafted the manuscript. FH participated in data collection and bioinformatics analyses, RW contributed to molecular genetic analyses and data interpretation, conceived the study and participated in final revision of the manuscript. CK collected samples, participated in phenotyping, bioinformatic analyses, performed association studies, conceived the study and finally revised the manuscript. All authors read and approved the final manuscript.

\section{Author details}

${ }^{1}$ Institute for Genome Biology, Leibniz Institute for Farm Animal Biology, 18196 Dummerstorf, Mecklenburg-Vorpommern, Germany. ${ }^{2}$ Faculty of Agricultural and Environmental Sciences, University of Rostock, 18059 Rostock, Germany. 


\section{Acknowledgements}

We thank our colleagues at the FBN Dummerstorf, who helped in the generation and care of the SEGFAM $\mathrm{F}_{2}$ resource population for their continuous support of our work. Skilful technical assistance of Simone Wöhl, Antje Lehmann, Marlies Fuchs, Oda Haufft, Bärbel Pletz and Emma Schröder is thankfully acknowledged. The study was supported by the Deutsche Forschungsgemeinschaft (DFG). The publication of this article was funded by the Open Access fund of the Leibniz Association.

\section{Competing interests}

The authors declare that they have no competing interests.

Received: 7 July 2015 Accepted: 3 March 2016

Published online: 31 March 2016

\section{References}

1. Shimomura Y. Congenital hair loss disorders: rare, but not too rare. J Dermatol. 2012;39:3-10

2. Ayers JR, Leipold HW, Schalles R, Cole D. Pathological studies of crossrelated congenital hypotrichosis in cattle. Zent Vet A. 1989;36:447-52.

3. Schalles RR, Cundiff LV. Inheritance of the "rat-tail" syndrome and its effect on calf performance. J Anim Sci. 1999;77:1144-7.

4. Miller WH, Scott DW. Black-hair follicular dysplasia in a Holstein cow Cornell Vet. 1990:80:273-7.

5. Ostrowski S, Evans A. Coat-color-linked hair follicle dysplasia in Buckskin Holstein cows in Central California. Agri-Practice. 1989;10:12-3.

6. Klungland H, Vage DI, Gomez-Raya L, Adalsteinsson S, Lien S. The role of melanocyte-stimulating hormone $(\mathrm{MSH})$ receptor in bovine coat color determination. Mamm Genome. 1995:6:636-9.

7. Olson T. Genetics of color variation. In: Fries R, Ruvinsky A, editors. The genetics of cattle. Wallingford: CABI; 1999. p. 33-53

8. Hecht B. Sequence analysis of Pmel17 as candidate gene for causing rattail syndrome in cattle. Master thesis, Brigham Young University. 2006

9. Jolly RD, Wills JL, Kenny JE, Cahill Jl, Howe L. Coat-colour dilution and hypotrichosis in Hereford crossbred calves. N Z Vet J. 2008;56:74-7.

10. Kuehn C, Weikard R. An investigation into the genetic background of coat colour dilution in a Charolais $\times$ German Holstein F2 resource population. Anim Genet. 2007;38:109-13.

11. Kühn C, Weikard R. Multiple splice variants within the bovine silver homologue (SILV) gene affecting coat color in cattle indicate a function additional to fibril formation in melanophores. BMC Genomics. 2007;8:335.

12. Marron B, Beever JE. A mutation in hephaestin-like 1 (HEPHL 1) is responsible for hypotrichosis in belted Galloway cattle. In: Proceedings of the plant and animal genome (PAG) 20th conference, 14-18 January, San Diego. 2012

13. Markey A. Mapping of monogenic and quantitative trait loci using a whole genome scan approach and single nucleotide polymorphism platforms. Ph.D. thesis. University of Illinois. 2013.

14. Daetwyler HD, Capitan A, Pausch H, Stothard P, Van Binsbergen R, Brondum RF, et al. Whole-genome sequencing of 234 bulls facilitates mapping of monogenic and complex traits in cattle. Nat Genet. 2014;46:858-65

15. Kuehn C, Bellmann O, Voigt J, Wegner J, Guiard J, Ender K. An experimental approach for studying the genetic and physiological background of nutrient transformation in cattle with respect to nutrient secretion and accretion type. Arch Anim Breed. 2002;45:317-30.

16. O'Connell JR, Weeks DE. PedCheck: a program for identification of genotype incompatibilities in linkage analysis. Am J Hum Genet. 1998:63:259-66.
17. Purcell S, Neale B, Todd-Brown K, Thomas L, Ferreira MA, Bender D, et al. PLINK: a tool set for whole-genome association and population-based linkage analyses. Am J Hum Genet. 2007;81:559-75.

18. Hanna LL, Sanders JO, Riley DG, Abbey CA, Gill CA. Identification of a major locus interacting with MC1R and modifying black coat color in an F-2 Nellore-Angus population. Genet Sel Evol. 2014;46:4

19. Seaton G, Hernandez J, Grunchec J, White I, Allen J, De Koning D, et al. GridQTL: a grid portal for QTL mapping of compute intensive datasets. In Proceedings of the 8th world congress on animal genetics applied to livestock production: 13-18 August; Belo Horizonte. 2006

20. Zimin AV, Delcher AL, Florea L, Kelley DR, Schatz MC, Puiu D, et al. A whole-genome assembly of the domestic cow, Bos taurus. Genome Biol. 2009;10:R42.

21. Abecasis GR, Cherny SS, Cookson WO, Cardon LR. Merlin-rapid analysis of dense genetic maps using sparse gene flow trees. Nat Genet. 2002;30:97-101.

22. Lander ES, Green P. Construction of multilocus genetic-linkage maps in Humans. Proc Natl Acad Sci USA. 1987:84:2363-7.

23. Kong A, Cox NJ. Allele-sharing models: LOD scores and accurate linkage tests. Am J Hum Genet. 1997:61:1179-88.

24. Kang HM, Sul JH, Service SK, Zaitlen NA, Kong SY, Freimer NB, et al. Variance component model to account for sample structure in genomewide association studies. Nat Genet. 2010;42:348-54.

25. Weikard R, Hadlich F, Kuehn C. Identification of novel transcripts and noncoding RNAs in bovine skin by deep next generation sequencing. BMC Genomics. 2013;14:789.

26. Annotation Bos taurus release 104. National Center of Biotechnology Information. 2015. ftp://ftp.ncbi.nih.gov/genomes/Bos taurus/GFF.

27. Schmutz SM, Moker JS, Clark EG, Shewfelt R. Black hair follicular dysplasia, an autosomal recessive condition in dogs. Can Vet J. 1998;39:644-6.

28. Welle M, Philipp U, Ruefenacht S, Roosje P, Scharfenstein M, Schuetz E, et al. MLPH genotype-melanin phenotype correlation in dilute dogs. J Hered. 2009;100:S75-9.

29. Drögemueller C, Philipp U, Haase B, Guezel-Apel AR, Leeb T. A noncoding melanophilin gene (MLPH) SNP at the splice donor of exon 1 represents a candidate causal mutation for coat color dilution in dogs. J Hered. 2007;98:468-73.

30. Basit S, Khan S, Ahmad W. Genetics of human isolated hereditary hair loss disorders. Clin Genet. 2015;88:203-12.

31. Seitz JJ, Schmutz SM, Thue TD, Buchanan FC. A missense mutation in the bovine MGF gene is associated with the roan phenotype in Belgian Blue and Shorthorn cattle. Mamm Genome. 1999:10:710-2.

32. Picardo M, Cardinali G. The genetic determination of skin pigmentation: KITLG and the KITLG/c-Kit pathway as key players in the onset of human familial pigmentary diseases. J Invest Dermatol. 2011;131:1182-5.

33. Guenther CA, Tasic B, Luo L, Bedell MA, Kingsley DM. A molecular basis for classic blond hair color in Europeans. Nat Genet. 2014;46:748-52.

34. Basit S, Wali A, Aziz A, Muhammad N, Jelani M, Ahmad W. Digenic inheritance of an autosomal recessive hypotrichosis in two consanguineous pedigrees. Clin Genet. 2011;79:273-81.

\section{Submit your next manuscript to BioMed Central and we will help you at every step:}

- We accept pre-submission inquiries

- Our selector tool helps you to find the most relevant journal

- We provide round the clock customer support

- Convenient online submission

- Thorough peer review

- Inclusion in PubMed and all major indexing services

- Maximum visibility for your research

Submit your manuscript at www.biomedcentral.com/submit
C Biomed Central 